\title{
Simultaneously Resettable Arguments of Knowledge
}

\author{
Chongwon $\mathrm{Cho}^{1}$, Rafail Ostrovsky ${ }^{12}$, \\ Alessandra Scafuro ${ }^{3}$, and Ivan Visconti ${ }^{3}$ \\ 1 Department of Computer Science, UCLA \\ 2 Department of Mathematics, UCLA, USA \\ \{ccho, rafail\}@cs.ucla.edu \\ 3 Dipartimento di Informatica, University of Salerno, Italy \\ \{scafuro,visconti\}@dia.unisa.it
}

\begin{abstract}
In this work, we study simultaneously resettable arguments of knowledge. As our main result, we show a construction of a constantround simultaneously resettable witness-indistinguishable argument of knowledge (simres $\mathcal{W} \mathcal{I}$ AoK, for short) for any NP language. We also show two applications of simres $\mathcal{W}$ IAoK: the first constant-round simultaneously resettable zero-knowledge argument of knowledge in the Bare Public-Key Model; and the first simultaneously resettable identification scheme which follows the knowledge extraction paradigm.
\end{abstract}

\section{Introduction}

Interaction and private randomness are the two fundamental ingredients in Cryptography. They are especially important for achieving zero-knowledge proofs [15]. In [7] Canetti, Goldreich, Goldwasser and Micali showed that when private randomness is limited and re-used in multiple instances of a proof system, it is still possible to preserve the zero-knowledge requirement. The setting proposed by [7] is of a malicious verifier that resets the prover, therefore forcing the prover to run several protocol executions using the same randomness. This setting applies to protocols where the prover is implemented by a stateless device. Therefore, a prover can only count on the limited (hardwired) randomness while it can be adaptively reset any polynomial number of times. The resulting security notion against such powerful verifiers is referred to as resettable zero knowledge $(r \mathcal{Z K})$ and is provably harder to achieve than concurrent zero knowledge [1118]. Feasibility results have been achieved in [717 in the standard model with the following round-complexity: polylogarithmic for $r \mathcal{Z K}$ and constant for resettable witness indistinguishability $(r \mathcal{W I}$, in short). Since then, it was also shown how to achieve resettable zero knowledge in the Bare Public-Key (BPK) model, introduced by Canetti et al. [7], where one can obtain better round complexity and assumptions [19:10 1 22 21]. Very recently, it has been shown [13 that resettable statistical zero knowledge for non-trivial languages is possible. 
The "reverse" of the above question has been considered by Barak, Goldreich, Goldwasser and Lindell [4 where a malicious prover resets a verifier, called resettable soundness. In [4, it has been shown how to obtain resettable soundness along with $\mathcal{Z K}$ in a constant number of rounds.

Barak et al. 4] proposed the challenging simultaneous resettability conjecture, where one would like to prove that a protocol is secure against both a resetting malicious prover and a resetting malicious verifier. The existing machinery turned out to be insufficient, and a definitive answer required almost a decade. In the work of Deng, Goyal and Sahai 9] they showed a resettably sound $r \mathcal{Z K}$ argument for NP with polynomial round complexity. Very recently, results in the BPK model for simultaneous resettability have been obtained in 812 with a constant number of rounds.

Arguments of knowledge under simultaneous resettability. Argument systems are often used with a different goal than proving membership of an instance in a language. Indeed, it is commonly required to prove knowledge (possession) of a witness instead of the truthfulness of a statement. Since arguments of knowledge serve as major building blocks in Cryptography (e.g., in identification schemes⿱11), it is an interesting question whether the previous results for arguments of membership extend to arguments of knowledge. Unfortunately, arguments of knowledge have been achieved so far only when one party can reset. That is, we have $\mathrm{r} \mathcal{Z K}$ arguments of knowledge [7] and, separately, resettably sound $\mathcal{Z K}$ arguments of knowledge [4]. Instead, when reset attacks are possible in both directions, no result is known even when only $r \mathcal{W I}$ with resettable argument of knowledge is desired.

It is important to note that resettable security for ZAPs comes almost for free because of the minimal round complexity ( 1 or 2 rounds). However, it is not known how to accommodate for knowledge extraction, unless one relies on non-standard (e.g., non-falsifiable) assumptions. For the case of resettably sound $\mathrm{r} \mathcal{Z K}$, all the above results $[9|8| 2]$ critically use an instance-dependent technique along with ZAPs: when the statement is true (i.e., when proving $r \mathcal{Z K}$ ), the prover/simulator can run ZAPs which allow the use of multiple witnesses. Such use of multiple witnesses gives some flexibility that turns out to be very useful to prove resettable zero knowledge. Instead, when the statement is false, the protocols are designed so that adversarial malicious prover must stick with some fixed messages during the execution of protocol. Therefore, rewinding capabilities do not help the resetting malicious prover since he can not change those fixed messages. This is critically used in the proofs of resettable soundness in order to reach a contradiction when a prover proves a false statement. It is easy to see that the above approach fails when arguments of knowledge are considered. Indeed, when the malicious resetting prover proves a true statement, the same freedom that allows one to prove $r \mathcal{Z K} / \mathrm{r} \mathcal{W} \mathcal{I}$, also gives extra power to the malicious prover. Consequently, designing an extractor appears problematic and new

${ }^{1}$ Bellare et al. in [5] gave various definitions for identification schemes when the adversary can also reset the proving device. 
techniques seem to be needed so that the simultaneous resettability conjecture is resolved even when we consider knowledge extraction.

Our results. Our main result is the first construction of a constant-round simultaneously resettable witness-indistinguishable argument of knowledge 2 (in short, simres $\mathcal{W}$ IAoK) for any NP language. Our protocol is based on the novel use of ZAPs and resettably sound zero-knowledge arguments, which improves over the techniques previously used in $[98$ ] as well as concurrent and independent work 3 of 16 .

We show several applications of our main result. First, we show that by combining two executions of our protocol for simres $\mathcal{W} \mathcal{I} A$ oK, we obtain a constantround simultaneously resettable zero-knowledge argument of knowledge in the BPK model. This improves the results of [82] which do not enjoy witness extraction with respect to adversarial resetting provers.

As another application of our main protocol, we also consider the question of secure identification under simultaneous resettability and show how to use the above simres $\mathcal{W} \mathcal{I}$ AoK to obtain the first simultaneously resettable identification scheme which follows the knowledge extraction paradigm. We describe it by extending the work of Bellare, et al. [5].

In addition, in the full version of this paper, we show how to obtain a constantround resettably sound concurrent zero knowledge argument of knowledge in the BPK model by relying on collision-resistant hash functions only (CRHFs, for short) (i.e., we do not require ZAPs, and thus trapdoor permutations).

Notation. We denote by $n \in \mathbb{N}$ the security parameter and by PPT the property of an algorithm of running in probabilistic polynomial-time. A function $\epsilon$ is negligible in $n$ (or just negligible) if for every polynomial $p(\cdot)$ there exists a value $n_{0} \in \mathbb{N}$ such that for all $n>n_{0}$ it holds that $\epsilon(n)<1 / p(n)$. We denote by $x \leftarrow \mathcal{D}$ the sampling of an element $x$ from the distribution $\mathcal{D}$. We also use $x \stackrel{\$}{\leftarrow}$ A to indicate that the element $x$ is sampled from set A according to the uniform distribution. Let $\mathcal{P}, \mathcal{V}$ be interactive Turing machines, we denote by $\langle\mathcal{P}(\cdot), \mathcal{V}(\cdot)\rangle(x)$ the random variable representing the local output of $\mathcal{V}$ when interacting with $\mathcal{P}$ where $x$ is the common input and the randomness of each machine is uniformly and independently chosen.

Blum's protocol. We will use the 3-round WIPoK protocol of Blum 6 for the NP-complete language Graph Hamiltonicity (HC) as main ingredient of our construction. We refer to Blum's protocol as BL and to BL1, BL2, BL3 its three rounds.

${ }^{2}$ In this work, we will never consider the case of resettable soundness along with non-resettable argument of knowledge. Therefore, each time we mention together resettable soundness and argument of knowledge, we mean that both soundness and witness extraction hold against a malicious resetting prover.

${ }^{3}$ In a very recent and independent work [16, Goyal and Maji achieved simultaneously resettable secure computation. Their work achieves (with simulation-based security) simultaneous resettability with polynomial round complexity assuming also the existence of lossy trapdoor encryption. 


\section{Resettably Sound $r \mathcal{W} \mathcal{I}$ Arguments of Knowledge}

Our goal is to obtain a construction that is resettably-sound resettable $\mathcal{W} \mathcal{I}$ and a resettable argument of knowledge in a constant number of rounds. The only known constant-round simultaneously-resettable $\mathcal{W} \mathcal{I}$ protocol is rZAP which is not an argument of knowledge and as discussed previously there is not much hope to transform it in an argument of knowledge (even without considering resettability).

A typical paradigm: determining message and consistency proof. Typically, protocols dealing with a resetting adversary ([4/9]) rely on the following paradigm: the resetting party is required to provide a special message (called determining message) that determines her own action for the rest of the protocol. Namely, for each protocol message the resetting party is required to prove that such message is consistent with the determining message (we call this proof a consistency proof). Moreover, the actual randomness used by the honest party in the protocol depends on the determining message (typically the honest party applies a pseudorandom function (PRF) on it). The combination of the randomness depending on the determining message and the consistency proof given by the resetting party, suppresses the resetting power of the adversary. Indeed, due to the consistency proof, the resetting party can not change a message previously played without first having changed the determining message (unless she is able to fake the consistency proof). However, if she changes the determining message, then the honest party plays the protocol with (computationally) fresh randomness (unless the pseudo-randomness of the PRF is violated). We will follow this paradigm to construct our simultaneously resettable witness indistinguishable argument of knowledge as well. Recall that as specified above, we do not know how to from rZAPs that are already simultaneously resettable and try to transform them in arguments of knowledge. Our starting point is Blum's proof of knowledge 6]. In the following discussion we show incrementally how to transform such protocol to enjoy resettable witness indistinguishability and resettable soundness (this transformation is already known in literature) to finally present our novel technique to obtain also resettable argument of knowledge.

Resettable $\mathcal{W I}$ and stand-alone argument of knowledge [4]. When the verifier can reset the prover, following the above paradigm, it is easy to construct a resettable $\mathcal{W} \mathcal{I}$ system starting from Blum's protocol. In Blum's protocol the only message from $\mathcal{V}$ to $\mathcal{P}$ is the challenge. The modified resettable version requires that $\mathcal{V}$ sends a statistically binding commitment of the challenge as determining message. The only other protocol message of $\mathcal{V}$ is the opening of the commitment which, due to the binding property, is itself a proof that the message is consistent with the determining message. Note that such modified protocol is no longer an argument of knowledge since the extractor has the same power of the malicious verifier. In order to allow only the extractor to cheat, the next step is to avoid the opening as a proof of consistency. Instead of the actual 
opening of the commitment, $\mathcal{V}$ is required to send the challenge along with a ressound (non-black-box) $\mathcal{Z} \mathcal{K}$ argument ([3]). The (non-black box) extractor can send an arbitrary challenge and prove consistency with the determining message by using the (stand-alone) non-black-box simulator (recall that only $\mathcal{V}$ might reset here). The resulting protocol is resettable $\mathcal{W I}$ and (stand-alone) argument of knowledge ( $\mathrm{r} \mathcal{W}$ IAoK for short) and it is known from [4].

We use a modified version of such protocol. We require that the commitment sent by the verifier is statistically hiding (instead of statistically binding), and we use the statistical zero-knowledge argument of knowledge of [20].

Achieving Resettable Soundness and Resettable Argument of Knowledge: existent solutions do not work. We now deal with the case in which also the prover can reset. By the BGGL compiler [4, we know that any constant-round publiccoin $\mathcal{W} \mathcal{I}$ argument system can be upgraded to resettable soundness by simply requiring the honest verifier to apply a $\mathrm{PRF}$ on the first message received from the prover. However, since our aim is to obtain simultaneous resettability, we need to start from the $r \mathcal{W I A o K}$ protocol shown before, which is not public coin. Thus, following the paradigm and the technique of [9], we require that as first message, $\mathcal{P}$ sends the commitment of the randomness that will be used in the protocol: this is the determining message. Then upon each protocol message $\mathcal{P}$ proves that the message is honestly computed using the randomness committed in the determining message: this is the consistency proof. Since we are now in the setting in which both parties can reset each other the consistency proof must be provided with a simultaneous resettable tool. For this purpose we use rZAPs that are constant-round simultaneously resettable $\mathcal{W I}$ proofs. We denote the theorem to be proved with rZAP as "consistency theorem", since $\mathcal{P}$ proves that a message is honestly computed and consistent with the randomness committed in the determining message.

The technical problem using rZAPs is that since guarantee $\mathcal{W} \mathcal{I}$, the theorem being proved is required to have more than one witness (note that the simultaneously resettable protocol of [9] can not be used here since we aim to a constant-round construction). Recall that we want to use rZAP to provide the proof of consistency with the determining message. If the determining message is a statistically binding commitment of the randomness, then there exists a unique opening, which implies the existence of only one witness. On the other hand, if we use a statistically hiding commitment, then any opening is a legitimate witness, the theorem is always true and the benefit of the determining message vanishes. The solution to overcome this problem is to change the theorem to be proved with rZAP so that it admits more than one witness.

In [9] the consistency theorem is augmented with the theorem " $x \in L$ " that we call "trapdoor theorem" recalling FLS paradigm [12] but with a different purpose. We call it trapdoor to stress out that it is an escape for the prover that can pass the consistency proof essentially having freedom to change messages among resets. Hence in 98 , along with each protocol message, $\mathcal{P}$ is required to 
prove that either the protocol message is computed honestly with the randomness committed in the determining message, (i.e., the "consistency theorem") or $x \in L$ (i.e., the "trapdoor theorem").

This solution can be seen as an instance-dependent technique. Indeed, it is easy to see that a malicious prover can play messages inconsistently with the determining message and still pass the consistency check, therefore exploiting its resetting power, only when $x \in L$. Instead, when proving soundness, since $x \notin L$, the trapdoor theorem is false, hence due to soundness of rZAPs, the malicious prover is forced to play according to the determining message therefore honestly following the protocol specifications.

Unfortunately, such an instance-dependent solution suffices to prove resettable soundness but fails completely when one would like to prove witness extraction (i.e., the argument of knowledge property). The reason is that, when proving witness extraction, we have to construct an extractor that works against any malicious prover, even one who uses the witness of the trapdoor theorem when proving consistency of the protocol messages. This possible behavior harms the extractor in two ways (recall that the witness can be computed from two distinct transcripts of Blum's protocol that have the same first message): 1) upon seeing the challenge of the verifier/extractor, $\mathcal{P}$ resets it and changes the first message of Blum's protocol according to the challenge; 2) $\mathcal{P}$ acts as a resetting verifier in the non-black-box $\mathcal{Z} \mathcal{K}$ protocol, therefore preventing the extractor to use the standalone non-black-box simulator. Even though this is not harmful for the soundness property (a malicious prover can perform this attack only when $x \in L$ ), this attack kills the existence of the extractor. Therefore the above construction is only resettable $\mathcal{W I}$ and resettable sound. Concluding, the instance-dependent technique of [9] inherently prevents the existence of any extractor. New ideas are required to solve the problem.

Achieving Resettable Argument of Knowledge: the new technique. We propose a new "trapdoor" theorem that forces the resetting prover to honestly follow the protocol regardless of whether $x \in L$ or not.

The idea is the following. We require $\mathcal{P}$ to run two parallel executions of the $\mathrm{rWI}$ I A K shown above, that we denote as subprotocols $\pi_{0}, \pi_{1}$. In the determining message, in addition to the commitment of the random tape that will be used to run each sub-protocol, we require that $\mathcal{P}$ commits to a single bit. Then, the trapdoor theorem in sub-protocol $\pi_{d}$ will be the following: " $d$ is the bit committed in the determining message". Since in the determining message there is only one bit committed (the other two are commitments of random tapes), due to the statistical binding property of the commitment, the trapdoor theorem is true in only one sub-protocol. Hence, in at least one of the sub-protocols the trapdoor theorem is false regardless of whether $x \in L$ or not, and in such sub-protocol $\mathcal{P}$ is forced to honestly follow the $\mathrm{rWIAoK}$ protocol, playing consistently with the determining message.

More specifically, the final protocol goes as follows. $\mathcal{P}$ first sends the determining message which consists of the statistically binding commitment of the random tapes that will be used in each sub-protocol and of a single bit. Each 
sub-protocol is augmented with rZAPs sent by $\mathcal{P}$ to $\mathcal{V}$ in which $\mathcal{P}$ proves consistency with the determining message. Therefore, in each sub-protocol $\pi_{d}$, along with each message of the $\mathrm{r} \mathcal{W}$ IAoK protocol, $\mathcal{P}$ provides a rZAP for the following compound theorem: either the message is honestly computed and consistent with the determining message, or $d$ is the bit committed in the determining message. Finally, the verifier will accept the proof if and only if both sub-protocol executions are accepting.

It is easy to see that any malicious prover can not escape from following the determining message in at least one of the subprotocols. Indeed, let $b$ be the bit committed in the determining message. If on one hand, in sub-protocol $\pi_{b}$, a malicious $\mathcal{P}$ is not forced to be honest and can then use the resetting power to prove any false theorem (indeed among resets $\mathcal{P}$ can change the protocol messages without changing the determining message), on the other hand, in sub-protocol $\pi_{\bar{b}}$, the trapdoor theorem is false, thus the only way to provide an accepting rZAP is to follow the honest behavior playing messages derived from the determining message. Therefore, in sub-protocol $\pi_{\bar{b}}$, the extractor is guaranteed that 1) for sessions starting with the same determining message, the first round of Blum's protocol does not change, so that playing with two distinct challenges yields the extraction of the witness; 2) the extractor can run the stand-alone non-black-box $\mathcal{Z} \mathcal{K}$ simulator without being detected. Hence we have the following: sub-protocol $\pi_{\bar{b}}$ is resettably-sound and resettable argument of knowledge, while sub-protocol $\pi_{b}$ is not sound. Note that in both sub-protocols, the resettable $\mathcal{W} \mathcal{I}$ property is still preserved.

\subsection{Formal Construction of simres $\mathcal{W}$ IAoK}

We formally describe how to build a constant-round simultaneously resettable $\mathcal{W I}$ AoK (simresWIAoK) starting from Blum's protocol (BL protocol). We denote by SHCom, a two-round statistically hiding commitment scheme. We denote by SBCom the commitment procedure of a non-interactive statistically binding commitment scheme. We denote by $c \leftarrow \operatorname{SBCom}(v, s)$ (resp. SHCom) the output of the commitment of the value $v$ computed with randomness $s$. We use the resettably-sound statistical (non-black-box) $\mathcal{Z} \mathcal{K}$ AoK of [20] that we denote by resSZK. In our construction, we require that $\mathcal{P}$, at each round of the protocol (except the last that is the opening of commitments as required by BL protocol), provides a proof that either the messages are honestly computed according to the randomness committed in the first round, or the "trapdoor" condition is satisfied. Formally, $\mathcal{P}$ provides rZAPs for the following $\mathbf{N P}$ languages (except the language $\Lambda_{\mathrm{SHCom}}$ that is proved only by $\mathcal{V}$ using resSZK protocol).

$\Lambda_{\mathrm{BL} 1}$ : correctness and consistency of the first round of Blum's protocol (BL1). A tuple $\left(x, m, \mathrm{c}_{r_{b}}, \mathrm{c}_{b}\right) \in \Lambda_{\mathrm{BL} 1}$ if there exist $\left(r_{b}, s_{b}\right)$ such that $\mathrm{c}_{r_{b}}=\operatorname{SBCom}\left(r_{b}, s_{b}\right)$ and $m$ is honestly computed according to BL1 for the graph $x$ using randomness $f_{r_{b}}\left(\mathrm{c}_{b}\right)$.

$\Lambda_{\mathrm{V}}$ : correctness and consistency of verifier's messages of the protocol resSZK. A tuple $\left(m_{P}, m_{V}, \mathrm{c}_{r_{b}}, \mathrm{c}_{b}\right) \in \Lambda_{\vee}$ if there exist $\left(r_{b}, s_{b}\right)$ such that $\mathrm{c}_{r_{b}}=$ 
$\mathrm{SBCom}\left(r_{b}, s_{b}\right)$ and $m_{V}$ is honestly computed according to the verifier's procedure of the protocol resSZK having in input prover's message $m_{P}\left(m_{P}\right.$ corresponds to the concatenation of all messages played by the prover so far) using randomness $f_{r_{b}}\left(c_{b}\right)$.

$\Lambda_{\text {trap }}$ : trapdoor theorem (true only for sub-protocol $b$ ). The pair $\left(\mathrm{c}_{s}, b\right) \in \Lambda_{\text {trap }}$ if there exists $s$ such that $\mathrm{c}_{s}=\mathrm{SBCom}(b, s)$.

$\Lambda_{\mathrm{SHCom}}$ : validity of the opening (proved by $\mathcal{V}$ ). The pair $\left(\mathrm{c}_{s}, m\right) \in \Lambda_{\mathrm{SHCom}}$ if there exists $s$ such that $\mathrm{c}_{s}=\operatorname{SHCom}(m, s)$. Note that for a statistically hiding commitment scheme, any pair $\left(\mathrm{c}_{s}, m\right)$ is actually in $\Lambda_{\mathrm{SHCom}}$. Nevertheless, $\mathcal{V}$ proves this theorem using the argument of knowledge resSZK.

Protocol simres $\mathcal{W}$ IAoK consists of two phases (see Fig. 1). In the first phase, $\mathcal{P}$ and $\mathcal{V}$ generate the random tapes that they will use to run the sub-protocols. $\mathcal{P}$ sends $\mathcal{V}$ the commitments $\mathrm{c}_{r_{0}}, \mathrm{c}_{r_{1}}$ of two random strings $r_{0}, r_{1}$ and the commitment $c_{s}$ of a random bit $b$. This message is the determining message on which $\mathcal{V}$ applies a PRF to generate a pseudo-random tape (to be used to execute the sub-protocols). The second phase consists of a parallel execution of $\pi_{0}$ and $\pi_{1}$ (see Fig. 2). $\mathcal{P}$ runs each sub-protocol on theorem $x$, randomness $r_{0}, r_{1}$, and the witnesses for computing the rZAPs as inputs (i.e., the opening of the commitments of the determining message). $\mathcal{V}$ runs each sub-protocol using the pseudo-random tapes determined by the determining message received from $\mathcal{P}$. Each sub-protocol is resettable $\mathcal{W I}$, while only one of the two sub-protocols is resettably-sound and a resettable AoK. Since $\mathcal{V}$ accepts the proof only if both executions are accepting, the final protocol is also a resettably-sound resettable AoK.

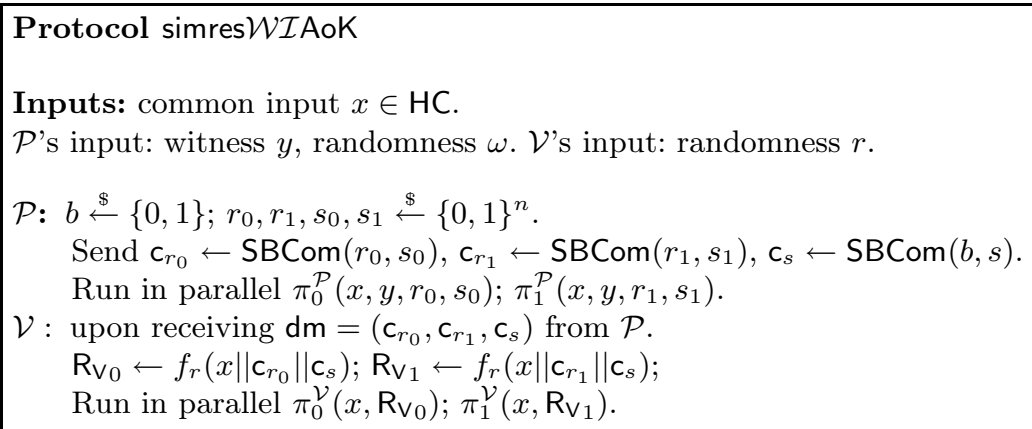

Fig. 1. Simultaneously Resettable Argument of Knowledge

The sub-protocol $\pi_{d}$ is described in Fig. 2. We omit the first round of the rZAP and the first round of the statistically hiding commitment scheme SHCom. rZAPs are computed with independent randomness. We stress out that the determining message for $\mathcal{V}$ is the first prover's message: $\mathrm{dm}=\left(\mathrm{c}_{r_{0}}, \mathrm{c}_{r_{1}}, \mathrm{c}_{s}\right)$. The determining message for $\mathcal{P}$ is the first verifier's message: $\left(c_{0}, c_{1}\right)$. 
Sub-protocol: $\pi_{d}=\left\langle\pi_{d}^{\mathcal{P}}\left(x, y, r_{d}, s_{d}\right), \pi_{d}^{\mathcal{V}}\left(x, \mathrm{R}_{\mathrm{V}_{d}}\right)\right\rangle$.

Inputs: common input: $x(\in \mathrm{HC})$. P's input: witness $y$ for $\mathcal{R}_{\mathrm{HC}}$; witness $\left(r_{d}, s_{d}\right)$ to prove $r Z A P$ 's consistency theorem. $\mathcal{V}$ 's input: randomness $\mathrm{R}_{\mathrm{V}_{d}}$. Protocols BL [6] and resSZK [20] are used as sub-protocols.

- V : Pick challenge for BL protocol: $c h_{d} \stackrel{\$}{\leftarrow}\{0,1\}^{n}$. Send $\mathrm{c}_{d} \leftarrow \operatorname{SHCom}\left(c h_{d}\right)$ to $\mathcal{P}$.

- $\mathcal{P}$ : upon receiving $\mathrm{c}_{d}$ (this is the determining message for $\mathcal{P}$ ):

1. Generate randomness $\mathrm{R}_{\mathrm{P}_{d}} \leftarrow f_{r_{d}}\left(x \| c_{d}\right)$.

2. Compute the step BL1 for the instance $x$ using randomness $\mathrm{R}_{\mathrm{P}_{d}}$. Let us denote the output as $\mathrm{mBL} 1^{d}$.

3. Send $\mathrm{mBL}^{d}$ to $\mathcal{V}$ along with the rZAP for theorem: $\left(\left(x, \mathrm{mBL} 1^{d}, \mathrm{c}_{r_{d}}, \mathrm{c}_{d}\right) \in\right.$ $\left.\Lambda_{\mathrm{BL} 1} \vee\left(\mathrm{c}_{s}, d\right) \in \Lambda_{\text {trap }}\right)$.

$-\mathcal{V}$ : if rZAP is accepting send $c h_{d}$ to $\mathcal{P}$.

Prove theorem $\left(\mathrm{c}_{d}, c h_{d}\right) \in \Lambda_{\mathrm{SHCom}}$ using resSZK protocol. Let $m_{\mathrm{P}_{\text {rszk }}}^{d}$ be the prover's message of sub-protocol resSZK (sent by $\mathcal{V}$ to $\mathcal{P}$ ) and $m_{\mathrm{V}_{\text {rszk }}}^{d}$ be the verifier's message of resSZK (sent by $\mathcal{P}$ to $\mathcal{V}$ ):

1. $(\mathcal{P} \rightarrow \mathcal{V})$ at each round of the protocol resSZK, upon receiving $m_{\mathrm{P}_{\mathrm{rszk}}}^{d}$ from $\mathcal{V}, \mathcal{P}$ computes $m_{\mathrm{V}_{\text {rszk }}}^{d}$ using randomness $\mathrm{R}_{\mathrm{P}_{d}}$ and sends $m_{\mathrm{V}_{\text {rszk }}}^{d}$ to $\mathcal{V}$ along with an rZAP for the theorem $\left(\left(m_{\mathrm{P}_{\mathrm{rszk}}}^{d}, m_{\mathrm{V}_{\mathrm{rszk}}}^{d}, \mathrm{c}_{r_{d}}, \mathrm{c}_{d}\right) \in \Lambda_{\mathrm{V}} \vee\left(\mathrm{c}_{s}, d\right) \in \Lambda_{\text {trap }}\right)$.

2. $(\mathcal{V} \rightarrow \mathcal{P})$ at each round of the protocol resSZK upon receiving $m_{\mathrm{V}_{\text {rszk }}}^{d}$ from $\mathcal{P}$, if rZAP is accepting $\mathcal{V}$ computes the next resSZK's prover message and sends it to $\mathcal{P}$. Otherwise it aborts.

- $\mathcal{P}$ : upon successfully completing the resSZK protocol compute step BL3 and send the message $\mathrm{mBL} 3^{d}$ to $\mathcal{V}$.

- If $\mathrm{mBL}^{d}$ is the correct third message of BL protocol $\mathcal{V}$ outputs accept, else outputs abort.

Fig. 2. Sub-protocol $\pi_{d}=\left(\pi_{d}^{\mathcal{P}}(\cdot), \pi_{d}^{\mathcal{V}}(\cdot)\right)$

\subsection{Security Proof}

In this section we provide the high-level proof of the simultaneous resettable witness indistinguishability property and the resettable argument of knowledge property of the protocol depicted in Fig. 1.

Resettable-soundness. Towards showing resettable soundness we start with the following observations. Recall that by $\mathrm{dm}$ we denote the determining message sent by $\mathcal{P}^{*}$ in the first round consisting of the commitment of two random seeds and the commitment of a bit (let us call the bit committed $b$ ).

1. The randomness used by $\mathcal{V}$ depends on $\mathrm{dm}$. In a resetting attack, malicious prover $\mathcal{P}^{*}$ activates $\mathcal{V}$ by selecting theorem and randomness, denoted by $(x, j)$ which forces $\mathcal{V}$ to run with the same randomness $r_{j}$ among several executions. However, the randomness actually used by $\mathcal{V}$ at each session is determined by the output of the PRF on seed $r_{j}$ and input $(x, \mathrm{dm})$. Thus, even if activated with the same random tape $r_{j}$, when receiving a new determining message, $\mathcal{V}$ executes the protocol with a fresh pseudo-random tape. 
Note that, due to the computational indistinguishability of the PRF, soundness holds against a computationally bounded adversary.

2. In sub-protocol $\pi_{b}$, the resetting power of $\mathcal{P}^{*}$ is effective since $\mathcal{P}^{*}$ can honestly prove the trapdoor theorem of the rZAP. Therefore, $\mathcal{P}^{*}$ is not forced to use the randomness committed in the determining message among multiple resetting attacks. Specifically, $\mathcal{P}^{*}$ can mount the following attack. $\mathcal{P}^{*}$ initiates a session labelled by $(x, j, \mathrm{dm})$. In the sub-protocol $\pi_{b}$, upon the reception of challenge $c h_{b}$ from $\mathcal{V}, \mathcal{P}^{*}$ resets $\mathcal{V}$ (while keeping the same determining message) back to the second round (the point after $\mathcal{V}$ has sent the commitment of the challenge). Then, $\mathcal{P}^{*}$ changes the message $\mathrm{mBL} 1^{b}$ according to the challenge $c h_{b}$ previously seen. This is possible using the trapdoor theorem, therefore $\mathcal{P}^{*}$ does not need to stick with the randomness committed in the determining message. Since the determining message is the same as before the reset, $\mathcal{V}$ will use the same challenge in the sub-protocol $\pi_{b}$. Thus, in this sub-protocol, $\mathcal{P}^{*}$ can prove any theorem by obtaining the challenge in advance and thus $\pi_{b}$ is not resettable sound.

3. In sub-protocol $\pi_{\bar{b}}$, the trapdoor theorem is always false, thus resetting $\mathcal{V}$ is ineffective. Indeed, in order to provide an accepting transcript, $\mathcal{P}^{*}$ must provide an rZAP that only exists when the "consistency" theorem is true, that is, each of $\mathcal{P}^{*}$ 's message is honestly computed according to the randomness committed in the determining message. By the statistically binding property of SBCom (there exists only one opening for the commitments $c_{s}$ and $c_{r_{\bar{b}}}$ ) and the soundness of rZAP (any unbounded $\mathcal{P}^{*}$ cannot prove a false theorem), $\mathcal{P}^{*}$ must be consistent with the randomness committed in the determining message. Therefore, $\pi_{\bar{b}}$ is resettably sound.

Assume that there exists a PPT malicious prover $\mathcal{P}^{*}$ and a pair $(x, j)$ such that $\mathcal{V}$ accepts $x$ with non-negligible probability for some $x \notin \mathrm{HC}$. By observation 1 , such a transcript is indexed by determining message $\mathrm{dm}$. Thus, the accepting transcript can be labelled by triple $(x, j, \mathrm{dm})$. By observation 2 , for the same determining message $\mathrm{dm}$, there are polynomially many distinct transcripts for sub-protocol $\pi_{b}\left(\mathcal{P}^{*}\right.$ can reset $\mathcal{V}$ polynomially many times and change the protocol messages). All these (partial) transcripts of $\pi_{b}$ can be accepting for $x \notin \mathrm{HC}$ since soundness does not hold for $\pi_{b}$. However, by observation 3 , for a fixed triple $\left(x, r_{j}, \mathrm{dm}\right)$, there exists only one possible accepting transcript for sub-protocol $\pi_{\bar{b}}$ since $\mathcal{P}^{*}$ is forced to honestly follow the BL protocol according to the randomness committed in the determining message. Therefore the soundness of BL is preserved when $\mathcal{P}^{*}$ resets $\mathcal{V}$ in $\pi_{\bar{b}}$. Since $\mathcal{V}$ accepts if and only if the executions of both sub-protocols are accepting, protocol simres $\mathcal{W} \mathcal{I}$ AoK is resettably sound.

Resettable argument of knowledge. To prove resettable argument of knowledge we show an expected PPT extractor that extracts the witness from any malicious prover $\mathcal{P}^{*}$ with probability that is negligibly close to the probability that $\mathcal{P}^{*}$ convinces an honest verifier. Let $(x, j, \mathrm{dm})$ be the label of the session in which $\mathcal{P}^{*}$ provides an accepting proof. The goal of the extractor is to obtain two accepting transcripts with the same BL1 message and two distinct challenges (for at least one sub-protocol) for the same label. 
Our extractor consists of two phases. In the first phase it follows the honest verifier procedure. When $\mathcal{P}^{*}$ has completed its execution, if there exists an accepting session labeled by $(x, j, \mathrm{dm})$ that we call "target session", the extractor proceeds to the second phase. In the second phase, the extractor obtains a distinct accepting transcript for the target session by cheating in the "opening" of the commitment by sending a challenge that is distinct from the one sent in the first phase and simulating the zero knowledge proof given by the verifier.

The crucial step of this phase is to detect the sub-protocol in which $\mathcal{P}^{*}$ is stuck with the randomness committed in $\mathrm{dm}$ and must follow the protocol honestly. Indeed, in such sub-protocol, the extractor can use the stand-alone simulator and open the statistically hiding commitment to any challenge. Note that the non-black-box simulator of the protocol resSZK takes as input the code of the malicious verifier. Thus, in order to use the simulator, the extractor must carefully prepare a machine which internally handles the interaction with $\mathcal{P}^{*}$ and forwards to the simulator only the messages belonging to the resSZK protocol played in one of the sub-protocol. One of the tasks of such machine is detecting the sub-protocol in which $\mathcal{P}$ is forced to be honest. Once the right sub-protocol has been detected, by the statistically-hiding property of SHCom, and by the statistical zero-knowledge property of protocol resSZK run by $\mathcal{V}$ instead of the opening, we are guaranteed that upon each rewind, $\mathcal{P}^{*}$ provides another accepting transcript for the target session with the same probability of the first phase. Finally, by the proof of knowledge property of Blum's protocol, collecting two distinct transcripts allows the extractor to compute the witness. The actual extractor requires an intermediate estimation step (as shown in [14]) in which the probability of having another accepting transcript for the label $(x, j, \mathrm{dm})$ is estimated. More details on the formal description of the extractor, the augmented machine and the formal proof can be found in the full version of this work.

Resettable witness indistinguishability. Recall that the protocol mainly consists of a single message from $\mathcal{P}$ to $\mathcal{V}$, the determining message $\left(c_{r_{0}}, c_{r_{1}}, c_{s}\right)$, and the parallel execution of $\pi_{0}$ and $\pi_{1}$. Such protocol can be seen as a parallel repetition of $\left(\Pi_{0}, \Pi_{1}\right)$ where $\Pi_{b}$ is the protocol $\pi_{b}$ augmented with the message $\left(\mathrm{c}_{s}, \mathrm{c}_{r_{b}}\right)$ sent from $\mathcal{P}$ to $\mathcal{V}$ and $b=0,1$.

Assume that there exists a resetting PPT distinguisher $\mathcal{V}^{*}$ for $\left(\Pi_{0}, \Pi_{1}\right)$. That is, $\mathcal{V}^{*}$ distinguishes whether $\mathcal{P}$ runs both protocols using witnesses sampled from distribution $Y_{0}=\left\{\bar{y}^{0}(\bar{x})\right\}_{\bar{x}}$ or from distribution $Y_{1}=\left\{\bar{y}^{1}(\bar{x})\right\}_{\bar{x}}$. Let us denote by $H_{0,0}$ the experiment in which $\mathcal{P}$ uses witnesses sampled from $Y_{0}$ when running both protocols $\left(\Pi_{b}, \Pi_{\bar{b}}\right)$, where $b$ is the bit committed in $c_{s}$, and by $H_{1,1}$ the experiment in which $\mathcal{P}$ uses witnesses sampled from $Y_{1}$ in both $\left(\Pi_{b}, \Pi_{\bar{b}}\right)$. We prove by hybrid arguments that experiments $H_{0,0}$ and $H_{1,1}$ are computationally indistinguishable. Let $n$ denote the number of theorems and $t$ the bound on the prover's random tapes. Consider the following hybrids.

$H_{1,0}$ : In this hybrid, in each session, $\mathcal{P}$ uses witnesses sampled from $Y_{1}$ to run protocol $\Pi_{b}$ and the bit $b$ is committed in the determining message in such session. The only difference between experiment $H_{1,0}$ and $H_{0,0}$ is in the 
witness used in $\Pi_{b}$. Assume that there exists a distinguisher between hybrids $H_{0,0}$ and $H_{1,0}$ then it is possible to construct an adversary $\mathcal{V}_{\mathrm{BL}}^{*}$ for the $\mathcal{W I}$ property of sub-protocol $\mathrm{BL}$ of $\Pi_{b}$. Note that, when $b$ is the bit committed in the determining message, the trapdoor theorem is true in $\Pi_{b}$. $\mathcal{V}_{\mathrm{BL}}^{*}$, on input $\left(\bar{x}, Y_{0}, Y_{1}\right)$, runs $\mathcal{V}^{*}$ as sub-routine and honestly executes the protocol $\Pi_{\bar{b}}$ using the witness belonging to $Y_{0}$. Instead for the execution of $\Pi_{b}$ it forwards the messages received from $\mathcal{V}^{*}$ and belonging to BL protocol to the external prover, while it simulates the remaining messages belonging to $\Pi_{b}$. The first difficulty in such reduction seems to be the fact that $\mathcal{V}^{*}$ can mount a reset attack asking the prover of $\Pi_{b}$ to run with the same randomness while changing the challenge of $\mathrm{BL}$ protocol. Instead, $\mathcal{V}_{\mathrm{BL}}^{*}$ can only mount a concurrent attack against the external BL's prover. Nevertheless, $\mathcal{V}_{\mathrm{BL}}^{*}$ can replicate the same attack of $\mathcal{V}^{*}$ for the following reasons. The randomness of the honest prover executing protocol $\Pi_{b}$ is computed on the determining message (the commitment of BL's challenge) received from $\mathcal{V}^{*}$. Due to the pseudo-randomness of PRF, when $\mathcal{V}^{*}$ changes the determining message the prover of $\Pi_{b}$ plays with fresh randomness. By the resettablysound argument of knowledge property of the resSZK protocol and by the computational binding property of SHCom we have that $\mathcal{V}^{*}$ can not maintain the same determining message and query the prover with two distinct BL's challenges. Thus the resetting power is suppressed and $\mathcal{V}_{\mathrm{BL}}^{*}$ can replicate the same attack as $\mathcal{V}^{*}$. The second difficulty is that for each protocol message the honest prover of $\Pi_{b}$ is required to send a rZAP proving that the messages are consistent with the randomness committed in the determining message. However, in the reduction $\mathcal{V}_{\mathrm{BL}}^{*}$ forwards the messages received by an external prover of BL's protocol, therefore it can not prove the consistency with the determine message. Nevertheless, since we are in the case in which the trapdoor theorem is true, $\mathcal{V}_{\mathrm{BL}}^{*}$ can forward the external messages and computes the rZAPs using the witness of the trapdoor theorem. Due to the resettable $\mathcal{W I}$ property of rZAP such deviation from the honest prover is not detected by any PPT $\mathcal{V}^{*}$. Then, by the $\mathcal{W} \mathcal{I}$ of BL protocol hybrids $H_{0,0}$ and $H_{1,0}$ are computationally indistinguishable.

$H_{0,1}^{i, j}$ (with $1 \leq i \leq n, 1 \leq j \leq t$ ): In hybrid $H_{0,1}^{i, j}$, in session $(i, j), \mathcal{P}$ runs protocol $\Pi_{\bar{b}}$ using the witness sampled from $Y_{1}$, while protocol $\Pi_{b}$ is run by using a witness sampled from $Y_{0}$, and $b$ is the bit committed in the determining message of such session. The only difference between experiment $H_{0,1}^{i, j}$ and $H_{0,1}^{i-1, j-1}$ is that in experiment $H_{0,1}^{i, j}$, in session $(i, j)$, the witness is sampled from $Y_{1}$ in the sub-protocol where the trapdoor theorem is false. Note that $H_{0,1}^{0,0}=H_{1,0}$. Assume that there exists a distinguisher between $H_{0,1}^{i, j}$ and $H_{0,1}^{i-1, j-1}$ then it is possible to construct an adversary for the hiding of the commitment scheme SBCom. The reduction works as follows. $\mathcal{A}$ playing in the hiding experiment obtains the challenge commitment $C$. Then it runs $\mathcal{V}^{*}$ as sub-routine and simulates the honest prover $\mathcal{P}$ as in experiment $H_{0,1}^{i-1, j-1}$, except that in session $(i, j)$ it proceeds as follows. It computes $\mathrm{c}_{r_{0}}, \mathrm{c}_{r_{1}}$ as the honest prover, while it sets $\mathrm{c}_{s}=C$, and sends the first round to $\mathcal{V}^{*}$. Then 
$\mathcal{A}$ uniformly chooses a bit $b$ and executes the protocol $\pi_{b}$ using a witness sampled from distribution $Y_{1}$ and protocol $\pi_{\bar{b}}$ using the witness sampled from distribution $Y_{0}$. Note that $\mathcal{A}$ can run both sub-protocols without knowing the opening of $C$ since also the honest $\mathcal{P}$ never uses such witness in the protocol execution. When $\mathcal{V}^{*}$ terminates its execution, $\mathcal{A}$ hands the output of $\mathcal{V}^{*}$ to the distinguisher and outputs whatever the distinguisher outputs. If $C$ is a commitment of $b$ then the experiment simulated by $\mathcal{A}$ is distributed identically to experiment $H_{0,1}^{i-1, j-1}$. Else if $C$ is a commitment of $\bar{b}$ then the experiment is distributed as experiment $H_{0,1}^{i, j}$. By the computational hiding of SBCom we have that experiments $H_{0,1}^{i, j}$ and $H_{0,1}^{i-1, j-1}$ are computational indistinguishable.

$H_{1,1}$ : In this hybrid, $\mathcal{P}$ uses a witness sampled from $Y_{1}$ to run protocol $\Pi_{b}$ and the bit $b$ is committed in the determining message. The only difference between experiment $H_{0,1}^{n, t}$ and experiment $H_{1,1}$ is in the witness used to run sub-protocol $\Pi_{b}$. By the same arguments put forth in proving the indistinguishability of hybrid $H_{1,0}$ and $H_{0,0}$, experiments $H_{0,1}^{n, t}$ and $H_{1,1}$ are computational indistinguishable. This completes the proof.

Theorem 1. If trapdoor permutations and collision-resistant hash functions exist, then the protocol shown in Fig. 1 is a Simultaneously Resettable Witness Indistinguishable Argument of Knowledge.

\section{Application in the BPK Model}

Here we show that by combining two instances of simres $\mathcal{W} \mathcal{I} A$ oK we obtain the first constant-round simultaneously resettable $\mathcal{Z K}$ AoK (simres $\mathcal{Z} \mathcal{K} A \circ K$ ) in the BPK model.

High-level overview of protocol and proof. The construction is very simple since it takes advantage of the properties guaranteed by the protocol simres $\mathcal{W}$ AAoK. We use it twice, once for a proof given by the verifier and once for a proof given by the prover. First, the verifier uses simres $\mathcal{W} \mathcal{I}$ AoK to prove knowledge of its secret key (one out of two possible sets of pre-images of a OWF), then the prover commits to its witness and finally uses simres $\mathcal{W} \mathcal{I}$ AoK to prove that the committed message is either a witness for the theorem $x \in L$ or a secret key. The intuition of why the protocol works is the following. First of all, the secret key of the verifier is protected by the one-wayness of the OWF, by the $r \mathcal{W I}$ property of the simres $\mathcal{W} \mathcal{I} A$ oK given by the verifier and by the resettable argument of knowledge of the simres $\mathcal{W} \mathcal{I}$ AoK given by the prover. Indeed, we will be able to prove that the witness extracted from the proof given by the prover can only be a witness for $x \in L$, otherwise we break either the hardness of the OWF or the $r \mathcal{W} \mathcal{I}$ property of simres $\mathcal{W} \mathcal{I}$ AoK. Instead, the security for the prover comes from the existence of a simulator against any resetting verifier. Indeed, we can design a simulator as follows: the simulator starts a main thread that is always updated with new messages until the simulator is stuck. This event happens when 
the simulator is supposed to commit to a witness and to then play the second simres $\mathcal{W} \mathcal{I}$ AoK. At this point, the simulator suspends the main thread and starts some rewinding threads in order to extract the secret key used by the adversarial verifier in that session. Once this is done, the simulator continues the main thread since it is not stuck anymore (i.e., it can simply commit to the extracted secret and use it as witness in the second simres $\mathcal{W} \mathcal{I} A$ oK). Since the number of identities of possible verifiers in the BPK model is polynomially bounded, we have that the simulator has to start only an expected polynomial number of rewinding threads, and thus its expected running time is polynomial. The indistinguishability of the view comes from the hiding of the commitment scheme and the $r \mathcal{W I}$ property of the second simres $\mathcal{W} \mathcal{I} A$ oK. Instead the resettable argument of knowledge of the first simres $\mathcal{W} \mathcal{I}$ AoK (i.e., the one given by the verifier) is helpful for guaranteeing the expected running time of the simulator. The commitment played in between the two executions of the simres $\mathcal{W} \mathcal{I}$ AoK plays an important role in breaking a possible malleability attack of the malicious sender.

The formal description of the protocol is provided in Fig. 3. For underlying primitives, we use a non-interactive statistically binding commitment scheme, denoted by SBCom, and a one-way function $g:\{0,1\}^{*} \rightarrow\{0,1\}^{*}$. In the protocol we use the following two NP relations: 1) a pair $((y, g), x) \in \mathcal{R}_{\Lambda_{\mathrm{ow}}}$ if $x$ is such that $y=g(x) ; 2)$ a pair $((c, m), r) \in \mathcal{R}_{\mathrm{SBCom}}$ if the string $r$ is such that $c=$ $\operatorname{SBCom}(m, r)$.

Theorem 2. If trapdoor permutations and collision-resistant hash functions exist, then protocol simres $\mathcal{Z} \mathcal{K}$ AoK is a constant-round simultaneously resettable zero-knowledge argument of knowledge in the BPK model.

For lack of space, the formal proof can be found in the full version of this paper.

\section{Simultaneously Resettable Identification Schemes}

In this section, we present the second application of our main protocol, the first construction of a simultaneously resettable identification scheme. Identification schemes represent one of the most successful practical applications of cryptographic protocols. The basic goal of an identification scheme is to prevent an adversary $\mathcal{A}$ from impersonating a honest user $\mathcal{P}$ to another honest user $\mathcal{V}$. However, this is not sufficient for some applications. Indeed, consider the case in which $\mathcal{V}$ provides a service to $\mathcal{P}$, and the service is restricted only to a small community controlled by $\mathcal{V}$. Then, $\mathcal{P}$ could give to another party $T$ that is not in the small community, some partial information about his secret that is sufficient for $T$ to obtain the service from $\mathcal{V}$, while still $T$ does not know $\mathcal{P}$ 's secret. The proof of knowledge property allows us to do secure identification as well as preventing the attack described above. When the identification protocol is a proof of knowledge, the sole fact that $T$ convinces $\mathcal{V}$ is sufficient to claim that one can extract the whole secret from $T$. This implies that $T$ obtained $\mathcal{P}$ 's secret key corresponding to his identity, and this is unlikely to happen in scenarios where the 


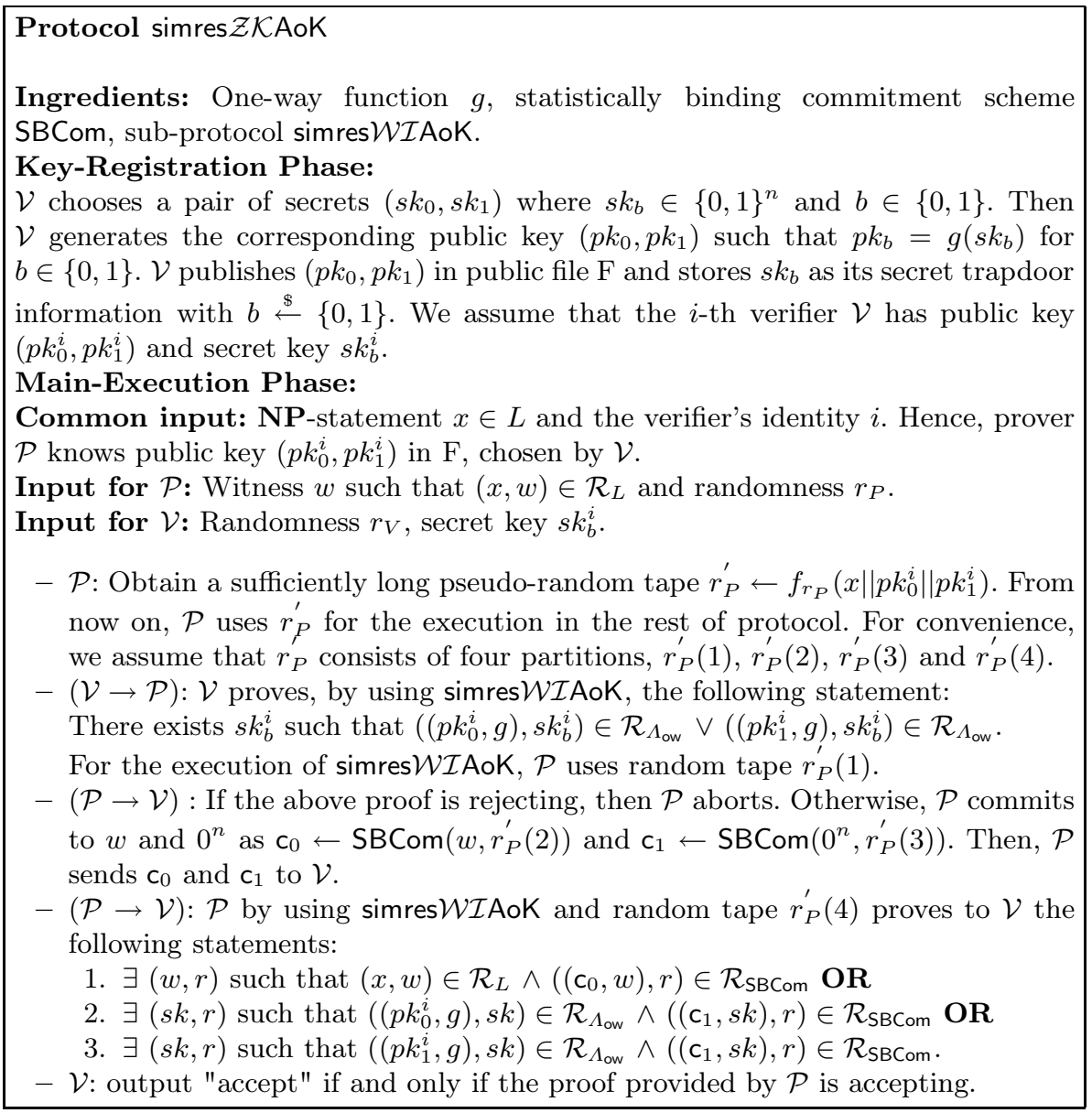

Fig. 3. Constant-Round Simultaneously Resettable $\mathcal{Z K A}$ oK in the BPK Model

same secret key is used for other critical tasks such as digital signatures. As discussed in the introduction, our simultaneously resettable identification scheme follows the above proof of knowledge paradigm. This extends the previous work of Bellare et al. [5] to a setting in which every party can be reset. We emphasize that our simultaneously resettable identification scheme is easily obtained from our main protocol simres $\mathcal{W} \mathcal{I} A$ oK, so achieving a constant round complexity.

Identification protocols secure against reset attacks. We introduce the notion of Reset-Reset-1 security as a generalization of the Concurrent-Reset-1 CR1 notion introduced in 5 . CR1 considers an adversary $I$, called impersonator, that plays in two phases. In the first phase, it interacts with a prover as a resetting verifier (Reset phase). In the second phase, it has no access to the prover anymore, but it tries to impersonate such a prover to an honest verifier (Concurrent phase). In the second phase, $I$ is not allowed to reset the verifier. In our new definition 
Reset-Reset-1 (RR1) the impersonator is allowed to reset in both phases. The formal definition is a straightforward extension of the one given in [5] and can be found in the full version of this work.

The protocol $\mathcal{I D}$. Let $f:\{0,1\}^{n} \rightarrow\{0,1\}^{*}$ be a one-way function, let $n$ be the security parameter. The public key of $\mathcal{P}$ is the pair $\left(\mathrm{pk}_{0}, \mathrm{pk}_{1}\right)$, the secret key is $x_{d}$ for a randomly chosen bit $d$, such that $\mathrm{pk}_{0}=f\left(x_{d}\right) \vee \mathrm{pk}_{1}=f\left(x_{d}\right)$. The protocol simply consists in $\mathcal{P}$ running the simres $\mathcal{W}$ IAoK protocol with $\mathcal{V}$ to prove that it knows the preimage of either $\mathrm{pk}_{0}$ or $\mathrm{pk}_{1}$. Formally, let $\Lambda_{\mathcal{I D}}$ be the following language $\Lambda_{\mathcal{I D}}=\left\{\left(y_{0}, y_{1}\right)\right.$ : there exists $x \in\{0,1\}^{n}$ s.t. $y_{0}=f(x) \vee$ $\left.y_{1}=f(x)\right\}$, then the identification scheme consists of $\mathcal{P}$ proving the statement $\left(\mathrm{pk}_{0}, \mathrm{pk}_{1}\right) \in \Lambda_{\mathcal{I D}}$ using simres $\mathcal{W} \mathcal{I}$ AoK.

Theorem 3. If a constant-round simultaneously resettable $\mathcal{W}$ IAoK protocol exists and one-way functions exist, then the above protocol is constant-round and secure in the RR1 setting.

Proof. Let $p k=\left(\mathrm{pk}_{0}, \mathrm{pk}_{1}\right)$ be the public key of a player $\mathcal{P}$. Assume that there exists a PPT adversary $I$ playing the RR1 experiment, that succeeds in impersonating an honest $\mathcal{P}$ with non-negligible probability. This means that $I$ is able to prove to an honest $\mathcal{V}$ that her identity is $p k=\left(\mathrm{pk}_{0}, \mathrm{pk}_{1}\right)$. Then we show that $I$ can be used to construct an adversary against the one-wayness of $f$, or a distinguisher for the resettable $\mathcal{W} \mathcal{I}$ property of the simres $\mathcal{W} \mathcal{I}$ AoK protocol. The resettable argument of knowledge property of simres $\mathcal{W}$ IAoK protocol is crucial to put forth both reductions.

Recall that, in the RR1 game, $I$ plays the first phase interacting as a resetting verifier $\mathcal{V}^{*}$ with $\mathcal{P}$ and in the second phase interacts as resetting prover $\mathcal{P}^{*}$ with $\mathcal{V}$ trying to impersonate $\mathcal{P}$.

First we show an adversary $\mathcal{A}$ that breaks the one-wayness of $f$. $\mathcal{A}$ has in input a challenge $y$ that is the output of $f(x)$ for some unknown $x$. The reduction works as follows. $\mathcal{A}$ picks $d \in\{0,1\}, x_{d} \in\{0,1\}^{n}$ and computes $\mathrm{pk}_{d}=f\left(x_{d}\right)$ and $\mathrm{pk}_{\bar{d}}=y$. Then it runs $I$ as subroutine, in the first phase $\mathcal{A}$ simulates the honest prover playing the simres $\mathcal{W} \mathcal{I}$ AoK protocol with witness $x_{d}$. In the second phase, $\mathcal{A}$ simulates the honest verifier to $I$. If $I$ provides an accepting proof, then $\mathcal{A}$ runs the extractor of the simres $\mathcal{W} \mathcal{I}$ AoK protocol and, by the resettable argument of knowledge property, except with negligible probability, it obtains the witness used by $I$ in the proof. In order to run the extractor, $\mathcal{A}$ prepares an augmented machine that internally contains all messages belonging to the first phase so that they can be internally played with $I$, while the messages sent by $I$ in the second phase are forwarded to the extractor. Now note that during the extraction process the extractor rewinds the machine several times changing the protocol messages (of the second phase), therefore $I$ could change her messages accordingly. Note that however, since there is a separation between the first phase and the second phase, this does not require to re-play messages of the first phase. Since, by assumption $f$ is a one-way function, the probability that the witness extracted corresponds to a pre-image of $y$ is negligible. 
Now, assume that the witness extracted from $I$ is $x_{d}$. Then we can construct a distinguisher $\mathcal{A}_{\mathcal{W I}}$ for the resettable witness indistinguishability property of simres $\mathcal{W}$ IAoK. $\mathcal{A}_{\mathcal{W I}}$ works as follows. It computes $\mathrm{pk}_{0}=f\left(x_{0}\right), \mathrm{pk}_{1}=f\left(x_{1}\right)$ and activates an external prover for the simres $\mathcal{W} \mathcal{I}$ AoK protocol with inputs $\left(\left(\mathrm{pk}_{0}, \mathrm{pk}_{1}\right),\left(x_{0}, x_{1}\right)\right)$. In the first phase, when $I$ runs as a verifier, $\mathcal{A}_{\mathcal{W I}}$ forwards all messages to the external prover of the simres $\mathcal{W} \mathcal{I}$ AoK. In the second phase, when $I$ runs as a prover, $\mathcal{A}_{\mathcal{W I}}$ follows the procedure of the honest verifier. Then, if $I$ provides an accepting proof, then $\mathcal{A}_{\mathcal{W I}}$ runs the extractor of the simres $\mathcal{W} \mathcal{I}$ AoK protocol. Finally by the resettable argument of knowledge property, except with negligible probability, it obtains the witness used by $I$ in the proof, i.e. it obtains $x_{0}$ or $x_{1}$. Now notice that in the previous experiment, when we tried to invert the one-way function, the witness extracted corresponded to the one used in the first phase, while $I$ was verifying the proof. Since this second experiment is identical to the previous one, it is again true that the extracted witness corresponds to the one used by the prover. Since the prover now is the external prover of simres $\mathcal{W} \mathcal{I}$ AoK, we have that the above adversary $\mathcal{A}_{\mathcal{W I}}$ breaks the $\mathrm{r} \mathcal{W} \mathcal{I}$ property of simres $\mathcal{W} \mathcal{I}$ AoK. By the $\mathrm{r} \mathcal{W}$ I property of simres $\mathcal{W}$ IAoK, this event happens with negligible probability only and thus $I$ wins the RR1 game with negligible probability.

Acknowledgments. Research supported in part by NSF grants 0830803, 09165174, 1065276, 1118126 and 1136174, US-Israel BSF grant 2008411, B. John Garrick Foundation, IBM, OKAWA Foundation, Lockheed-Martin Corporation and the Defense Advanced Research Projects Agency through the U.S. Office of Naval Research under Contract N00014-11-1-0392. The views expressed are those of the authors and do not reflect the official policy or position of the Department of Defense or the U.S. Government. The work of the third and fourth authors has been done while visiting UCLA and is supported in part by the European Commission through the FP7 programme under contract 216676 ECRYPT II.

\section{References}

1. Alwen, J., Persiano, G., Visconti, I.: Impossibility and Feasibility Results for Zero Knowledge with Public Keys. In: Shoup, V. (ed.) CRYPTO 2005. LNCS, vol. 3621, pp. 135-151. Springer, Heidelberg (2005)

2. Arita, S.: A constant-round resettably-sound resettable zero-knowledge argument in the bpk model. Cryptology ePrint Archive, Report 2011/404 (2011), http://eprint.iacr.org/

3. Barak, B.: How to go beyond the black-box simulation barrier. In: FOCS, pp. 106-115 (2001)

4. Barak, B., Goldreich, O., Goldwasser, S., Lindell, Y.: Resettably-sound zeroknowledge and its applications. In: FOCS, pp. 116-125 (2001)

5. Bellare, M., Fischlin, M., Goldwasser, S., Micali, S.: Identification Protocols Secure against Reset Attacks. In: Pfitzmann, B. (ed.) EUROCRYPT 2001. LNCS, vol. 2045, pp. 495-511. Springer, Heidelberg (2001)

6. Blum, M.: How to Prove a Theorem So No One Else Can Claim It. In: Proceedings of the International Congress of Mathematicians, pp. 1444-1451 (1986) 
7. Canetti, R., Goldreich, O., Goldwasser, S., Micali, S.: Resettable zero-knowledge (extended abstract). In: STOC, pp. 235-244 (2000)

8. Deng, Y., Feng, D., Goyal, V., Lin, D., Sahai, A., Yung, M.: Resettable Cryptography in Constant Rounds - The Case of Zero Knowledge. In: Lee, D.H. (ed.) ASIACRYPT 2011. LNCS, vol. 7073, pp. 390-406. Springer, Heidelberg (2011)

9. Deng, Y., Goyal, V., Sahai, A.: Resolving the simultaneous resettability conjecture and a new non-black-box simulation strategy. In: FOCS, pp. 251-260. IEEE Computer Society (2009)

10. Di Crescenzo, G., Persiano, G., Visconti, I.: Constant-Round Resettable Zero Knowledge with Concurrent Soundness in the Bare Public-Key Model. In: Franklin, M. (ed.) CRYPTO 2004. LNCS, vol. 3152, pp. 237-253. Springer, Heidelberg (2004)

11. Dwork, C., Naor, M., Sahai, A.: Concurrent zero-knowledge. In: Proceedings of the 20th Annual ACM Symposium on Theory of Computing, STOC 1998, pp. 409-418. ACM (1998)

12. Feige, U., Lapidot, D., Shamir, A.: Multiple noninteractive zero knowledge proofs under general assumptions. SIAM J. Comput. 29(1), 1-28 (1999)

13. Garg, S., Ostrovsky, R., Visconti, I., Wadia, A.: Resettable Statistical Zero Knowledge. In: Cramer, R. (ed.) TCC 2012. LNCS, vol. 7194, pp. 494-511. Springer, Heidelberg (2012)

14. Goldreich, O., Kahan, A.: How to construct constant-round zero-knowledge proof systems for np. J. Cryptology 9(3), 167-190 (1996)

15. Goldwasser, S., Micali, S., Rackoff, C.: The knowledge complexity of interactive proof-systems. In: Proceedings of the 17th Annual ACM Symposium on Theory of Computing, STOC 1985, pp. 291-304. ACM (1985)

16. Goyal, V., Maji, H.K.: Stateless cryptographic protocols. In: FOCS 2011 (2011)

17. Kilian, J., Petrank, E.: Concurrent and resettable zero-knowledge in polylogarithmic rounds. In: Proceedings of the 33rd Annual ACM Symposium on Theory of Computing, STOC 2001, pp. 560-569. ACM (2001)

18. Kilian, J., Petrank, E., Rackoff, C.: Lower bounds for zero knowledge on the internet. In: Proceedings of 39th IEEE Conference on the Foundations of Computer Science, FOCS 1998, pp. 484-492 (1998)

19. Micali, S., Reyzin, L.: Soundness in the Public-Key Model. In: Kilian, J. (ed.) CRYPTO 2001. LNCS, vol. 2139, pp. 542-565. Springer, Heidelberg (2001)

20. Pass, R., Rosen, A.: New and improved constructions of non-malleable cryptographic protocols. In: Proceedings of the 37th Annual ACM Symposium on Theory of Computing, STOC 2005, pp. 533-542. ACM (2005)

21. Scafuro, A., Visconti, I.: On round-optimal zero knowledge in the bare public-key model. In: EUROCRYPT. LNCS. Springer, Heidelberg (2012)

22. Yung, M., Zhao, Y.: Generic and Practical Resettable Zero-Knowledge in the Bare Public-Key Model. In: Naor, M. (ed.) EUROCRYPT 2007. LNCS, vol. 4515, pp. 129-147. Springer, Heidelberg (2007) 